\title{
Montelukast in the management of allergic rhinitis
}

\author{
Jaime A Lagos \\ Gailen D Marshall \\ Division of Clinical Immunology \\ and Allergy, University of Mississippi \\ Medical Center, Jackson, MS, USA
}

Correspondence: Gailen D Marshall Division of Clinical Immunology and Allergy, University of Mississippi Medical Center, 2500 North State Street, Jackson, MS 39216-4505, USA

Tel + | ||-60|-8|5-5527

Fax + I II-60I-8I5-4770

Email gmarshall@medicine.umsmed.edu

\begin{abstract}
Allergic rhinitis is the most common atopic disorder seen in the outpatient clinic setting diagnosed by history, physical exam and objective testing. According to the Allergic Rhinitis and its Impact on Asthma (ARIA) document, it is classified by chronicity (intermittent or persistent), and severity which is based on symptoms and quality of life (mild, or moderate/severe). It has enormous socioeconomic costs and significant reduction in quality of life. Allergen avoidance should be implemented, particularly in children, to reduce level of exposure; unfortunately efforts are often inadequate. Montelukast, a novel medication, is an antagonist to the leukotriene receptor. It is nonsedating, dosed once daily, and has a safety profile similar in adults and children with approval down to 6 months of age. A review of the literature undoubtedly establishes montelukast as a viable alternative for the treatment of seasonal allergic rhinitis. Its benefits are equivalent to antihistamines, when used as monotherapy, but less than intranasal corticosteroids. The addition of an antihistamine to montelukast does appear to have added benefits and at times is reported to be equivalent to intranasal corticosteroids.
\end{abstract}

Keywords: allergic rhinitis, montelukast, management, drug therapy

\section{Introduction}

Allergic rhinitis (AR) is the most common atopic disorder affecting some 20 to 40 million people annually in the US, including $10 \%$ to $30 \%$ of adults and up to $40 \%$ of children (Dykewicz et al 1998). Many believe this to be a conservative estimate with about one third of persons burdened with allergic rhinitis not seeking formal medical care (McMenamin 1994). The prevalence of AR has continued to increase during the past decades from $10 \%$ in 1970 to $30 \%$ in 2000 with severity ranging from mild to debilitating (Dykewicz et al 1998; Nathan et al 1997). The morbidity associated with AR is profound; attributing to 3.4 million lost workdays yearly, diminished work productivity, and cognition (McMenamin 1994). This morbidity is not only attributable to symptoms but also soporific effects of sedating antihistamines purchased over-thecounter by many patients. Direct and indirect costs associated with treating AR are substantial and reported to be US\$5.3 billion in the year 1996 (Ray et al 1999).

The pathophysiology of AR is characterized by inflammation of the nasal mucous membranes because of a complex response to nasal allergen exposure. Nasal congestion, sneezing, nonpurulent rhinorrhea as well as pruritis of the eyes, palate, and nose characterize the symptoms experienced by patients. If severe, problems may include paranasal sinus pressure and pain or eustachian tube dysfunction described as ear popping and fullness (Dykewicz et al 1998). According to the Allergic Rhinitis and its Impact on Asthma (ARIA) document, AR should be classified by chronicity (intermittent or persistent), and severity which is based on symptoms and quality of life (mild, or moderate/severe) (Bousquet et al 2001) (Table 1). The terms "seasonal" and "perennial" AR were previously categorized as AR by the clinically significant 
Table I Revised classification of AR from ARIA guidelines. Copyright (C) 1992. Reproduced with permission from Corren J, Adinoff AD, Irvin CG. 1992. Nasal beclomethasone prevents the seasonal increase in bronchial responsiveness in patients with allergic rhinitis and asthma. J Allergy Clin Immunol, 90:250-6.

\section{Classification of allergic rhinitis}

I- "Intermittent" means that the symptoms are present:

- Less than 4 days a week,

- Or for less than 4 weeks.

2- "Persistent" means that the symptoms are present:

- More than 4 days a week,

- And for more than 4 weeks.

3- "Mild" means that none of the following items are present:

- Sleep disturbance,

- Impairment of daily activities, leisure and/or sport,

- Impairment of school or work,

-Troublesome symptoms.

4- "Moderate-severe" means that one or more of the following items are present:

- Sleep disturbance,

- Impairment of daily activities, leisure and/or sport,

- Impairment of school or work,

- Troublesome symptoms.

Abbreviations: AR, allergic rhinitis;ARIA, Allergic Rhinitis and its Impact on Asthma.

aeroallergen. Perennial AR is associated with year round and indoor allergens including mold spores, cockroaches, dust mite fecal particles, animal dander, and occupational exposure. Seasonal AR is commonly referred to as "hay fever", developing during a defined pollen season, and is usually intermittent, as a result of allergic reactions to outdoor aeroallergens including mold spores, and pollens of trees, grasses, and weeds that depend on wind for cross-pollination. Commonly there is an overlap of "perennial" and "seasonal" symptoms in some geographic regions which has resulted in decreased use and confusion regarding these terms.

In a sensitized person, allergens exposed to the nasal mucosa initiate the allergic response by degranulation of preformed and newly synthesized mediators from mast cells after the cross linkage of cell-bound immunoglobulin $\mathrm{E}$ (IgE). An early-phase response develops because of cysteinyl leukotrienes (CysLTs), prostaglandins, histamine, tryptase, neuropeptides, and cytokines resulting in sneezing, pruritis, rhinorrhea, and congestion. During the late-phase, congestion primarily develops in response to cytokines, mediator release, and the infiltration of inflammatory cells including basophils, eosinophils, neutrophils, mast cells, and mononuclear cells 4-6 hours after allergen exposure (White and Kaliner 1992; Togias 2000). These cells in turn also release mediators including CysLTs, histamine, kinins, and eosinophil-derived mediators which are responsible for recruitment, activation, and perpetuation of cellular infiltrate resulting in continued chronic nasal congestion during the late phase (Ledford and Lockey 1998).

On physical examination, patients classically can have pale nasal mucosae with swollen, edematous turbinates, and clear nasal secretions (rhinorrhea). A thorough history and physical are sufficient to make a preliminary diagnosis and initiate treatment. Demonstrating specific IgE antibodies by either skin testing or in vitro radioallergosorbent (RAST) testing to which a person has become sensitized may be necessary in patients with difficult to treat allergic rhinitis in order to provide optimal treatment. Current treatment approaches include nonpharmacologic measures to reduce exposure to offending allergens and pharmacotherapy that includes several different classes of drugs. Optimal therapeutic drug selection depends upon several factors including severity (mild, moderate, or severe) and duration (intermittent, persistent) of symptoms, patient adherence (which includes dosing frequency, willingness to accept the mechanism of delivery and cost) and the clinician assessment of which pharmacologic class best treats a specific patient since not all agents are equally effective in all patients.

Nasal provocation testing although more commonly used in research than in the clinical setting, is useful in diagnosing and confirming sensitivity in occupational rhinitis, evaluating discrepancies between history and test(s), and to test for nonspecific reactivity to stimuli commonly observed in allergic rhinitis (Corrado et al 1986; Gerth-van-Wijk and Dieges 1991; Malm et al 1999). One method of provocation is with adenosine monophosphate (AMP), which acts indirectly via primed airway mast cells causing degranulation and release of mediators and in this manner acts a surrogate for airway inflammation.

\section{Treatment}

Optimal treatment of allergic rhinitis depends upon several factors. The first involves avoidance of implicated allergens. This remains the cornerstone of treatment in allergic disease; however two Cochrane meta-analysis (Gotzsche et al 1998; Kilburn et al 2001) and an ARIA position paper have brought this into question (Custovic and van Wijk 2005). In adults, the general consensus is that physical and chemical methods used to control dust mite and pet allergen levels is not supported when used as single or even multiple interventions. Whereas in children, environmental control measures do offer benefit and should be implemented when appropriate (Bjornsdottir et al 2003; Custovic and van Wijk 
2005). Unfortunately, the effort to appropriately reduce levels of indoor allergens is often too difficult for patients to accomplish and even more difficult is the prevention of exposure to outdoor allergens.

Pharmacotherapy includes oral and intranasal $\mathrm{H} 1$ antihistamines, intranasal corticosteroids, oral and intranasal decongestants, intranasal anticholinergics, intranasal cromolyn and leukotriene receptor antagonists. Traditionally, the selection of medication has been dependant on severity, intermittent versus persistent $A R$, and physician preference. A recent study found therapy chosen by physicians in an ad hoc fashion to be less effective than therapy directed by specific guidelines (Bousquet et al 2003). Several guidelines have recently been published and include: Allergy Report, Joint Task Force on Practice Parameters for Rhinitis, European Academy of Allergology and Clinical Immunology, and ARIA (Dykewicz et al 1998; AAAAI 2000; van Cauwenberge et al 2000; Bousquet et al 2001). None of the guidelines recommend oral first-generation antihistamines as part of treatment for AR. Second generation $\mathrm{H} 1$ antihistamines are in general recommended for mild to moderate disease as first line therapy, but not when treating nasal congestion. Oral decongestants are indicated in combination with oral antihistamines for nasal congestion, but patients should be monitored for side effects including elevated blood pressure, palpitations, tremor, sleep disturbance and should be used with caution in patients with certain conditions, eg, arrhythmia, coronary heart disease, hypertension, hyperthyroidism, glaucoma, diabetes, and urinary dysfunction (Jordana et al 1996). Topically applied intranasal decongestants should be limited in use to less than 10 days because prolonged use may lead to tachyphylaxis (rhinitis medicamentosa), which is a rebound swelling of the nasal membranes or drug induced rhinitis (Graf et al 1995; Graf and Hallen 1996). Intranasal corticosteroids (long considered the "gold standard") are often required in moderate to severe disease and are effective in reducing all symptoms of allergic rhinitis in addition to eye symptoms associated with allergic conjunctivitis (Bernstein et al 2004; Singulair PI 2005). Mast cell stabilizers are regarded as safe, but are usually less effective than antihistamines and nasal corticosteroids expect, perhaps, in a prophylactic role. Intranasal anticholinergic sprays are recommended to reduce rhinorrhea and/or postnasal drainage not controlled by other medications. However, as a class, anticholinergics have few effects on other symptoms of AR. The other major therapeutic class of drug indicated for AR therapy is the leukotriene-receptor antagonist (LTRA) to which montelukast belongs. There were no comments in 4 of 5 guideline statements at time of the publications, which has led many in the health field to question its place in treatment. The guidelines did mention that antileukotriene medications, such as montelukast, may be of value in the treatment of AR and further testing was needed to fully evaluate efficacy. Research since the various guideline publications continues and there are now sufficient data to make the use of LTRA for the treatment of AR a reasonable option. This article is intended to inform physicians and other healthcare professionals about montelukast's pharmacology, efficacy, tolerability, and therapeutic role in the management of AR.

\section{Pharmacology}

Montelukast is an orally active, highly selective cysteinyl leukotriene type-1 receptor antagonist of leukotreine D4, with affinities approximately two-fold greater than the natural ligand. It is rapidly absorbed achieving peak plasma concentration $\left(\mathrm{C}_{\max }\right)$ in 3 to 4 hours and with a mean bioavailability of $64 \%$ following a $10 \mathrm{mg}$ oral administration. For the $5 \mathrm{mg}$ chewable, $\mathrm{C}_{\max }$ is achieved in 2 to 2.5 hours with mean bioavailability of $73 \%$ fasting versus $63 \%$ with a standard meal. Regarding the $4 \mathrm{mg}$ chewable, the mean $\mathrm{C}_{\max }$ is achieved in 2 hours, with fasting, for patients in 2 to 5 year old range. More than $99 \%$ is bound to plasma proteins with minimal distribution across the blood-brain barrier. Metabolism occurs via liver P450 (CYP) 3A4 and 2CP microsomes, with potent inhibition of P450 2C8. Excretion occurs almost exclusively in bile with a half-life from 2.7 to 5.5 hours in healthy adults. The pharmacokinetic profile is similar in females and males, young and elderly. $10 \mathrm{mg}$ is recommended for ages 15 and older, $5 \mathrm{mg}$ chewable tablets for patients 6 to 14 years of age, $4 \mathrm{mg}$ chewable in patients 2 to 5 years of age, and $4 \mathrm{mg}$ oral granule formulation in pediatrics for asthma in 12 to 23 months and AR in 6 to 23 months of age. In patients with mild to moderate hepatic insufficiency, no dosage adjustment is required but data are lacking regarding severe hepatic impairment. Because montelukast and its metabolites are almost exclusively excreted in bile and not urine, it has not been evaluated in patients with renal insufficiency. Drug interactions were noted to not to be clinically significant with theophylline, and did not change the pharmacokinetics profile of warfarin about prothrombin time or international normalized ratio (INR). Urinary excretion of digoxin is not altered and there is no change in the plasma concentration profile with terfenadine. Prolongation of QTc was not observed with coadministration of terfenadine (Markham and Faulds 1998; Singulair PI 2005). 


\section{Tolerability}

Montelukast is well tolerated and has a safety profile similar in pediatric and adult populations. In studies looking at safety and adverse effects, in general, there was no clinical or laboratory difference in adverse experiences versus placebo. Side effects most commonly reported above placebo included headache, otitis media, upper respiratory infection, and pharyngitis. These events occurred at a frequency of $\geq 2 \%$ above placebo (Markham and Faulds 1998; Singulair PI 2005). Night terrors, although not commonly reported, can develop.

\section{Efficacy and qualify of life}

The efficacy of montelukast in the treatment of seasonal AR has been studied quite extensively over the past few years as monotherapy, combined with a second generation antihistamine, and with or without intranasal corticosteroids. Several studies evaluating the efficacy of montelukast as monotherapy were large, double blind, placebo-controlled trials (Meltzer et al 2000; Nayak et al 2002; Philip et al 2002, 2004; van Adelsberg, Philip, LaForce, et al 2003; van Adelsberg, Philip, Pedinoff, et al 2003). Statistically significant endpoints in symptom scores compared with placebo included daytime nasal symptoms scores (in 5 of 6 studies), daytime eye symptom scores, nighttime symptom scores, and composite symptom scores. Nighttime symptoms (difficulty falling asleep, nighttime awakenings, and congestion upon awaking) appeared to have a better response with montelukast compared with antihistamines as reported in 3 separate studies (Philip et al 2002; Nayak et al 2002; van Adelsberg, Philip, LaForce, et al 2003).

In studies evaluating montelukast combined with ceterizine or loratadine versus monotherapy, the data are largely equivocal. In a study performed by Meltzer and colleagues (2000) the combination of montelukast and loratadine taken concomitantly provided a significant improvement in daily diary cards, Rhinoconjunctivitis Quality of Life Questionnaire (RQLQ), compared with placebo and each agent alone. In a second study (Kurowski et al 2004), the combination of montelukast with ceterizine did show additive benefits in achieving decreased scores when compared with both monotherapies while another study (Nayak et al 2002) showed no statistically significant symptom improvement with the combination treatment compared with either montelukast or loratadine alone. Even though no statistical significance was observed, there was a trend toward increased improvement of symptoms compared with monotherapy.

The data comparing intranasal corticosteroids with monotherapy of montelukast or antihistamine show nasal steroids to have a greater efficacy on daytime and nighttime symptoms (Pullerits et al 2002; Ratner et al 2003; Martin et al 2005). However, when intranasal corticosteroids are compared with combination therapy with montelukast and antihistamines, results vary depending on the antihistamine chosen (ie, cetirizine vs loratadine) and which intranasal corticosteroid was used (ie, fluticasone vs budesonide vs mometasone). In one study comparing fluticasone with a combination of montelukast and loratadine, both groups showed improvements in daytime nasal allergic symptoms compared with placebo, but fluticasone was superior in controlling nighttime symptoms (Pullerits et al 2002). Conversely, the combination of montelukast and cetirizine was as effective as intranasal mometasone in patients with allergic rhinitis, and intranasal budesonide in patients with seasonal AR and asthma (Wilson, Orr, et al 2001; Wilson, Sims, et al 2001). Lastly, further benefit is shown in patients already treated with intranasal corticosteroids and antihistamines when montelukast is added for uncontrolled symptoms of AR.

In addition to symptom scores, studies with RQLQ resulted in modest but significant improvements for both antihistamines and montelukast as monotherapy compared with placebo (Topuz and Ogmen 2003; Perry et al 2004; Patel et al 2005). Not all parameters responded equally with nonnose and non-eye symptoms being cited as not improving with montelukast (Perry et al 2004). Combination therapy resulted in greater benefits in reducing scores within all RQLQ domains as compared with single agent treatment (Perry et al 2004; Patel et al 2005).

The efficacy of montelukast in the treatment of perennial AR has not been studied as extensively as in seasonal AR. There was one large placebo controlled trial, with reported improvements in daytime nasal score symptoms, quality of life, as well as improvements in Global Evaluation of Allergic Rhinitis by patients (Patel et al 2005). A second study evaluating cat allergen exposure on lower and upper airways showed a trend in improvement in the primary endpoint of nasal symptoms score and significant benefit with nasal congestion during both allergen challenge and the recovery phase (Perry et al 2004). Additionally, analysis of simultaneous lower and upper airway responses showed more patients were protected from both asthma and rhinitis with montelukast compared with placebo (Phipatanakul et al 2002).

\section{Conclusions}

Montelukast belongs to a unique class of drugs that specifically target the cysteinyl leukotriene receptor 1 . A review of the literature supports the claim of montelukast as a viable 
alternative for the treatment of seasonal AR. Its benefits are generally equivalent to antihistamines when used as monotherapy regarding efficacy and quality of life improvement, but less than intranasal corticosteroids. The addition of an antihistamine to montelukast does appear to have added benefits and may be equivalent to intranasal corticosteroids, at least in some patients. More research needs to be completed in order to determine montelukast's true efficacy in treating perennial AR, but early studies appear favorable.

Treatment with montelukast is slightly more complicated than asking the question "where does montelukast belong in therapy" as there are many factors a physician needs to consider in selecting a medication. Severity, perennial versus seasonal and associated comorbidities all should be considered. Cost is another factor often overlooked by those prescribing medications. In treating mild AR, either montelukast or a second generation antihistamine may be chosen because of comparable efficacy. If no improvement is appreciated, changing to ICS may generally be more effective than adding of a second medication. The use of an inhaled corticosteroid (ICS) as first line treatment is also acceptable in mild disease. When disease progresses to a moderate or severe classification, most professionals agree that ICS remains the first line agent in treatment of AR. In those patients with less than optimal improvement on ICS alone, careful questioning about regular use of the product is extremely important. Add on therapy with a 2nd generation antihistamine may often be a better choice particularly in those who will not or cannot use the ICS regularly as prescribed. The selection of the antihistamine is not based on relevant data but from a financial perspective because second generation antihistamines are now available over the counter and in generic form and therefore more cost effective. However, the 2 nd and even 3 rd generation antihistamines are not completely devoid of sedating effects (except fexofenadine) and should therefore be carefully considered if prescribed for a patient. In those who continue with symptoms despite double therapy, Topuz and colleagues (2003) showed about $10 \%$ of patients will benefit with the addition of montelukast. Lastly, the selection of montelukast will also depend on comorbid conditions. This is exemplified by the coexistence of AR in up to $78 \%$ of patients with asthma having coexisting $A R$, which contrasts with the prevalence in the general population of $20 \%$. Likewise $3 \%-5 \%$ of asthmatics in the general population have AR compared with $19 \%-38 \%$ of patients with allergic rhinitis having coexisting asthma. The united airway has been proposed to explain these associations supported in part by the continuous respiratory mucosa, include the occurrence of bronchial hyperreactivity following nasal provocation test in ARs without asthma, and improvement in asthma control after treatment of AR with intranasal corticosteroids (Welsh et al 1987; Corren et al 1992; Corren 1997). Likewise, selecting montelukast in this group of patients is beneficial because of its proven efficacy in both conditions. Again this needs to be adjusted on an individual patient basis because of the severity of asthma and AR. Patients may require intranasal corticosteroids in addition to antileukotrienes and antihistamines. Further studies need to be performed in order to fully delineate the optimal treatment strategies regarding pharmacotherapy. The guidelines do not infer preference of medications at this time, but only instruct us in the stepping up and down of treatment.

\section{References}

[AAAAI] American Academy of Allergy, Asthma, and Immunology. 2000. The allergy report [online]. Accessed 22 November 2005. URL: http://www.aaaai.org/ar/volume1.pdf.

Bernstein DI, Levy AL, Hampel FC, et al. 2004. Treatment with intranasal fluticasone propionate significantly improves ocular symptoms in patients with seasonal allergic rhinitis. Clin Exp Allergy, 34:952-7.

Bjornsdottir US, Jakobinudottir S, Runarsdottir V, et al. 2003. The effect of reducing levels of car allergen (Fel d 1) on clinical symptoms in patients with cat allergy. Ann Allergy Asthma Immunol, 91:189-94.

Bousquet J, Lund VJ, van Cauwenberge P, et al. 2003. Implementation of guidelines for seasonal allergic rhinits:a randomized controlled trial. Allergy, 58:733-41.

Bousquet J, van Cauwenberge P, Khaltaev N, et al. 2001. Allergic rhinitis and its impact on asthma. J Allergy Clin Immunol, 108:S147-334.

Corrado OJ, Gould CA, Kassab JY, et al. 1986. Nasal response of rhinitic and non-rhinitic subjects to histamine and methacholine: a comparative study. Thorax, 41:863-8.

Corren J, Adinoff AD, Irvin CG. 1992. Nasal beclomethasone prevents the seasonal increase in bronchial responsiveness in patients with allergic rhinitis and asthma. J Allergy Clin Immunol, 90:250-6.

Corren J. 1997. Allergic rhinitis and asthma: how important is the link? J Allergy Clin Immunol, 99:S781-6.

Custovic A, van Wijk RG. 2005. The effectiveness of measures to change the indoor environment in the treatment of allergic rhinitis and asthma : ARIA update (in collaboration with GA2LEN). Allergy, 60:1112-15.

Dykewicz MS, Fineman S, Skoner DP, et al. 1998. Diagnosis and management of rhinitis: complete guidelines of the Joint Task Force on Practice Parameters in Allergy, Asthma and Immunology. Ann Allergy Asthma Immunol, 81:478-518.

Gerth-van-Wijk R, Dieges PH. 1991. Nasal hyper-responsiveness to histamine, methacholine and phentolamine in patients with perennial nonallergic rhinitis and in patients with infectious rhinitis. Clin Otolaryngol, 16:133-7.

Gotzsche PC, Hammarquist C, Burr M. 1998. House dust mite control measures in the management of asthma : meta-analysis. BMJ, 317:1105-10; discussion, 1110.

Graf P, Hallen H, Juto JE. 1995. Four-week use of oxymetazoline nasal spray (Nezeril) once daily at night induces rebound swelling and nasal hyperreactivity. Acta Otolaryngol, 115:71-5.

Graf P, Hallen H. 1996. Effect on the nasal mucosa of long-term treatment with oxymetazoline, benzalkonium chloride, and placebo nasal sprays. Laryngoscope, 106:605-9.

Jordana G, Dolovich J, Briscoe MP, et al. 1996. Intranasal fluticasone propionate versus loratadine in the treatment of adolescent patients with seasonal allergic rhinitis. J Allergy Clin Immunol, 97:588-95. 
Kilburn S. Lasserson TJ. McKean M. 2001. Pet allergen control measures for allergic asthma in children and adults. Cochrane Database Syst Rev, 1:CD002989.

Kurowski M, Kuna P, Gorski P. 2004. Montelukast plus ceterizine in the prophylactic treatment of seasonal allergic rhinitis: influence on clinical symptoms and nasal allergic inflammation. Allergy, 59:280-8.

Ledford DK, Lockey RF. 1998. Allergic rhinitis: Understanding the process (a major contributor to health problems-and on the rise). J Respir Dis, 19:576-84.

Malm L, Gerth-van-Wijk R, Bachert C. 1999. Guildelines for nasal provocations with aspects on nasal patency, airflow, and airflow resitance. Rhinology, 37:133-5.

Markham A, Faulds D. 1998. Montelukast. Drugs, 56:251-6.

Martin B, Andrews CP, van Bavel JH, et al. 2005. Comparison of fluticasone propionate aqueous nasal spray and oral montelukast for the treatment of seasonal allergic rhinitis symptoms. Ann Allergy Asthma Immunol, 96:851-7.

McMenamin P. 1994. Costs of hay fever in the United States in 1990. Ann Allergy, 73:35-9.

Meltzer EO, Malmstrom K, Lu S, et al. 2000. Concomitant montelukast and loratadine as treatment for seasonal allergic rhinitis: a randomized, placebo-controlled clinical trial. J Allergy Clin Immunol, 105:917-22.

Nathan RA, Meltzer EO, Selner JC, et al. 1997. Prevalence of allergic rhinitis in the United States. J Allergy Clin Immunol, 99:S808-S14.

Nayak AS, Philip G, Lu S, et al. 2002. Efficacy and tolerability of montelukast alone or in combination with loratadine in seasonal allergic rhinitis: a multicenter, randomized, double blind, placebo controlled trial performed in the fall. Ann Allergy Asthma Immunol, 88:592-600.

Patel P, Philip G, Yang W, et al. 2005. Randomized, double-blind, placebocontrolled study of montelukast for treating perennial allergic rhinitis. Ann Allergy Asthma Immunol, 95:551-7.

Perry TT, Corren J, Philip G, et al. 2004. Protective effect of montelukast on lower and upper respiratory tract responses to short-term cat allergen exposure. Ann Allergy Asthma Immunol, 93:431-8.

Philip G, Malmstrom K, Hampel FC, et al. 2002. Montelukast for treating seasonal allergic rhinitis: a randomized, double blind, placebo-controlled trial performed in the spring. Clin Exp Allergy, 32:1020-8.

Philip G, Nayak A, Berger W, et al. 2004. The effect of montelukast on rhinitis symptoms in patients with asthma and seasonal allergic rhinitis. Curr Med Res Opin, 10:1549-58.

Phipatanakul W, Nowak-Wegrzyn A, Eggleston PA, et al. 2002.The efficacy of montelukast in the treatment of cat allergen-induced asthma in children. J Allergy Clin Immunol, 109:794-9.
Pullerits T, Praks L, Ristioja V, et al. 2002. Comparison of a nasal glucocorticoid, antileukotriene, and a combination of antileukotriene and antihistamine in the treatment of seasonal allergic rhinitis. $J$ Allergy Clin Immunol, 109:949-55.

Ratner PH, Howland WC, Arastu R, et al. 2003. Fluticasone propionate aqueous nasal spray provided significantly greater improvement in daytime and nighttime nasal symptoms of seasonal allergic rhinitis compared with montelukast. Ann Allergy Asthma Immunol, 109:536-42.

Ray NF, Baraniuk JN, Thamer M, et al. 1999. Direct expenditures for the treatment of allergic rhinoconjunctivitis in 1996, including the contributions of related airway illnesses. J Allergy Clin Immunology, 103:401-7.

Singulair PI. 2005. Singulair prescribing information. Whitehouse Station, NJ: Merck \& Co. Inc., June 1998-2005.

Togias A. 2000. Unique mechanistic features of allergic rhinitis. $J$ Allergy Clin Immunol, 90:599-604.

Topuz B, Ogmen GG. 2003. Montelukast as an adjuvant to mainstay therapies in patients with seasonal allergic rhinitis. Clin Exp Allergy, 33:823-6.

van Adelsberg J, Philip G, LaForce CF, et al. 2003. Randomized controlled trial evaluating clinical benefit of montelukast for treating spring seasonal allergic rhinitis. Ann Allergy Asthma Immunol, 90:214-22.

van Adelsberg J, Philip G, Pedinoff AJ, et al. 2003. Montelukast improves symptoms of seasonal allergic rhinitis over a 4-week treatment period. Allergy, 58:1268-76.

van Cauwenberge P, Bachert C, Passalacqua G, et al. 2000. Consensus statement on the treatment of allergic rhinitis. European Academy of Allergology and Clinical Immunology. Allergy, 55:116-34.

Welsh PW, Stricker WE, Chu CP, et al. 1987. Efficacy of beclomethasone nasal solution, flunisolide, and cromolyn in relieving symptoms of ragweed allergy. Mayo Clin Proc, 62:125-34.

White MV, Kaliner MA. 1992. Mediators of allergic rhinitis. J Allergy Clin Imтипо , 90:699-704.

Wilson AM, Orr LC, Sims EJ, et al. 2001. Effects of monotherapy with intranasal corticosteroid or combined oral histamine and leukotriene receptor antagonists in seasonal allergic rhinits. Clin Exp Allergy, 31:61-8.

Wilson AM, Sims EJ, Orr LC, et al. 2001. Effects of topical corticosteroids and combined mediator blockade on domiciliary and laboratory measurements of nasal function in seasonal allergic rhinitis. Ann Allergy Asthma Immunol, 87:344-9. 